\title{
A cognitive behavioral therapy intervention to reduce fear of hypoglycemia in young adults with type 1 diabetes (FREE): study protocol for a randomized controlled trial
}

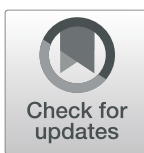

Pamela Martyn-Nemeth ${ }^{1 *}$ D, Jennifer Duffecy ${ }^{2}$, Laurie Quinn ${ }^{1}$, Chang Park ${ }^{3}$, Dan Mihailescu ${ }^{4}$ and Sue Penckofer ${ }^{5}$

\begin{abstract}
Background: In persons with type 1 diabetes (T1D), hypoglycemia is the major limiting factor in achieving optimal glycemic control. All persons with T1D are at risk for hypoglycemia (blood glucose level $<70 \mathrm{mg} / \mathrm{dl}$ ), which is lifethreatening and accompanied by serious physical and psychological symptoms, resulting in profound fear of hypoglycemia $(\mathrm{FOH})$ and reduced quality of life. Young adults with T1D are at risk for $\mathrm{FOH}$ and have worse glycemic control and self-management behavior than other age groups with T1D. FOH also results in increased glycemic variability (GV). A major gap exists in how to manage FOH. Our overall objective is to reduce $\mathrm{FOH}$ and improve diabetes self-management, glycemic control, and GV in young adults with T1D to reduce or delay diabetes complications and improve quality of life. We aim to (1) determine the feasibility and acceptability of an eight-week cognitive behavioral therapy (CBT)-based Fear Reduction Efficacy Evaluation (FREE) intervention in young adults with T1D who experience FOH; and (2) determine the impact of the FREE intervention, compared to an attention control group, on the outcomes $\mathrm{FOH}$, self-management, glycemic control (A1C), and glycemic variability (continuous glucose monitoring recordings).

Methods/design: A randomized controlled trial in 50 young adults aged 18 to 35 years with T1D will be used. Eligible subjects will be randomized to the intervention program (Fear Reduction Efficacy Evaluation [FREE]) or attention control group. A one-week run-in phase is planned, with baseline measures of $\mathrm{FOH}$, self-management behavior, A1C, and real-time continuous glucose monitoring recordings (RT-CGM) to calculate GV for both groups. The intervention group will participate in eight weekly individual one-hour sessions using CBT and exposure treatment for specific fears. RT-CGM and a daily FOH diary will be used as feedback cues as part of the FREE program. The attention control group will participate in eight weekly individual one-hour diabetes selfmanagement education (DSME) sessions and wear a RT-CGM device (to measure GV only) over 8 weeks. At completion, FOH will be measured, and RT-CGM recordings will be analyzed to determine differences between the FREE and control groups.
\end{abstract}

Discussion: Findings from this proposed pilot study will serve as the foundation for a larger trial to reduce $\mathrm{FOH}$ and improve self-management, glycemic control, and GV.

Trial registration: ClinicalTrials.gov: A cognitive behavioral therapy (CBT) intervention to reduce fear of hypoglycemia in type 1 diabetes, NCT03549104. Registered June 7, 2018

Keywords: Fear of hypoglycemia, Type 1 diabetes, Cognitive behavioral therapy, Randomized controlled trial

\footnotetext{
* Correspondence: pmartyn@uic.edu

'Department of Biobehavioral Health Science, University of Illinois at Chicago

College of Nursing, 845 South Damen Avenue (MC 802), Chicago, IL 60612,

USA

Full list of author information is available at the end of the article
}

(c) The Author(s). 2019 Open Access This article is distributed under the terms of the Creative Commons Attribution 4.0 International License (http://creativecommons.org/licenses/by/4.0/), which permits unrestricted use, distribution, and reproduction in any medium, provided you give appropriate credit to the original author(s) and the source, provide a link to the Creative Commons license, and indicate if changes were made. The Creative Commons Public Domain Dedication waiver (http://creativecommons.org/publicdomain/zero/1.0/) applies to the data made available in this article, unless otherwise stated. 


\section{Background}

In persons with type 1 diabetes (T1D), iatrogenic hypoglycemia is the major limiting factor in achieving optimal blood glucose control [1]. All persons with T1D are at risk for hypoglycemia (blood glucose level $<70$ $\mathrm{mg} / \mathrm{dl}$ [2]), which is life-threatening [3] and has serious physical and psychological sequelae. One psychological sequela is fear of hypoglycemia $(\mathrm{FOH})[4,5]$. FOH can be incapacitating, causing panic [4], anxiety [6], phobic disorders [7], and greatly diminished quality of life (QOL) [8].

Despite advances in insulin therapy and glucosesensing technology, $\mathrm{FOH}$ remains a critical deterrent to T1D self-management, psychological well-being, and QOL [9]. Fear is conceptualized as an emotion arising from a cognitive appraisal of a specific threat or danger [10]. Normal fear is adaptive, stimulating more vigilance and improved performance; heightened fear leads to increased anxiety and may result in a delay to action or inappropriate action [10-12]. Fear may mimic the symptoms of hypoglycemia and impair its detection, exacerbating the problem $[5,13]$. At the extreme, fears can develop into anxiety disorders and phobias [7, 11, 14].

Previous negative experiences of hypoglycemia influence diabetes self-management behaviors [9, 15-20]. Diabetes self-management is defined as the knowledge, skills, and behaviors needed for diabetes self-care [21]. Insulin doses may be inappropriately reduced and diet may be modified to avoid hypoglycemia. Dietary modifications may include excessive eating, particularly more carbohydrates [22, 23] or snacking at night [19, 24]. These modifications lead to increased glycemic variability (GV; the intra-day fluctuations in blood glucose) and poor glycemic control. Registry data from the T1D Exchange Clinic Registry revealed that only $13 \%$ of young adults achieved glycemic targets [25], and, in a survey of self-management practices, $45 \%$ of young adults reported that they did not reach their glycemic goals due to $\mathrm{FOH}$ [9].

Young adulthood is the developmental period from age 18 to 35 years, when individuals transition to independent diabetes care, as well as establish independence, careers, family, and parenthood [26]. This is before the onset of long-term diabetes complications, when healthy behavior changes can have a critical impact on future health [27].

Over the past three decades, newer technologies have been designed to help patients with diabetes manage their treatment regimens, including continuous glucose monitoring (CGM) systems, insulin pumps, sensoraugmented pump therapy with insulin suspend features, and insulin bolus calculators. While these have improved glucose control (i.e., A1C), improved A1C has not consistently translated into reduced FOH [28-34]. Diabetes education programs typically discuss $\mathrm{FOH}$ but have limited strategies to manage it [4]. Glucose management and blood glucose awareness training have had variable effects on FOH [4, 35-43]. Outcomes demonstrated that, as glucose levels lower, worry levels do not consistently decrease [43]. We hypothesize that the lack of consistent and sustainable reductions in $\mathrm{FOH}$ has occurred because the focus of these programs has been on glycemic control, not FOH.

$\mathrm{GV}$ has been associated with more frequent episodes of hypoglycemia [44] and fluctuations between glucose extremes (i.e., hypo- to hyperglycemic), which may occur with overtreatment of a hypoglycemic episode. Evidence supports the role that GV plays in generation of oxidative stress [45], endothelial dysfunction [46], and diabetes complications in T1D [47]. GV has also been associated with increased risk for cardiovascular events [63]. Though A1C provides a biomarker for average blood glucose over a 2- to 3-month period, it does not capture daily blood glucose fluctuations (Fig. 1). Individuals may have an optimal A1C yet high GV [48]. GV is influenced by self-management behavior [49] and amenable to change with appropriate intervention.

The proposed study will pilot test a cognitive behavioral therapy (CBT)-based program to decrease $\mathrm{FOH}$ and examine its association with GV. Heightened fear develops from memories of previous negative hypoglycemic events that create a conditioned negative response to future fear triggers $[11,50]$. The conditioned fear response is reframed through cognitive restructuring of negative thoughts, regulation of emotions (FOH), and changing maladaptive behaviors (self-management behavior). Exposure therapy will be used to reduce FOH that is out of proportion to the threat through habituation to previously fearful situations (Fig. 2) [51]. Realtime continuous glucose monitor (RT-CGM) readings and fear diary review will be feedback cues to reinforce learning. The goal is not to replace standard diabetes therapy, but improve diabetes self-management [52] through reducing fear. The CBT intervention will be compared to usual diabetes education currently in place to address self-management behavior.

The specific aims for this study are:

In 50 young adults with T1D (18-35 years) [26] who experience $\mathrm{FOH}$, we will:

1. Determine the feasibility and acceptability of an eight-week CBT-based Fear Reduction Efficacy Evaluation (FREE) intervention

2. Determine the impact of the FREE intervention, compared to an attention control group, on the outcomes $\mathrm{FOH}$, self-management, glycemic control (A1C), and glycemic variability (RT-CGM recordings) 

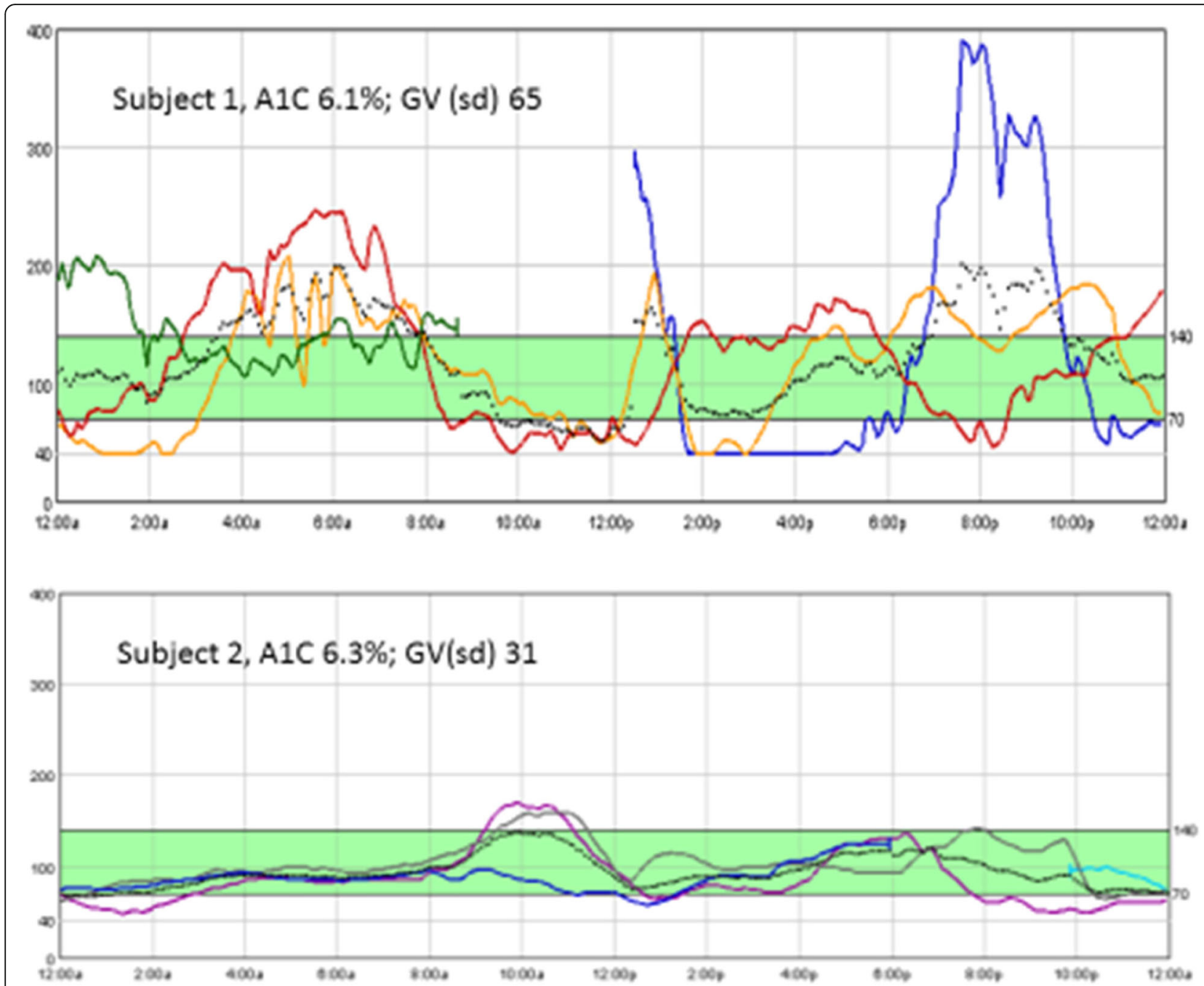

Fig. 1 Glycemic variability

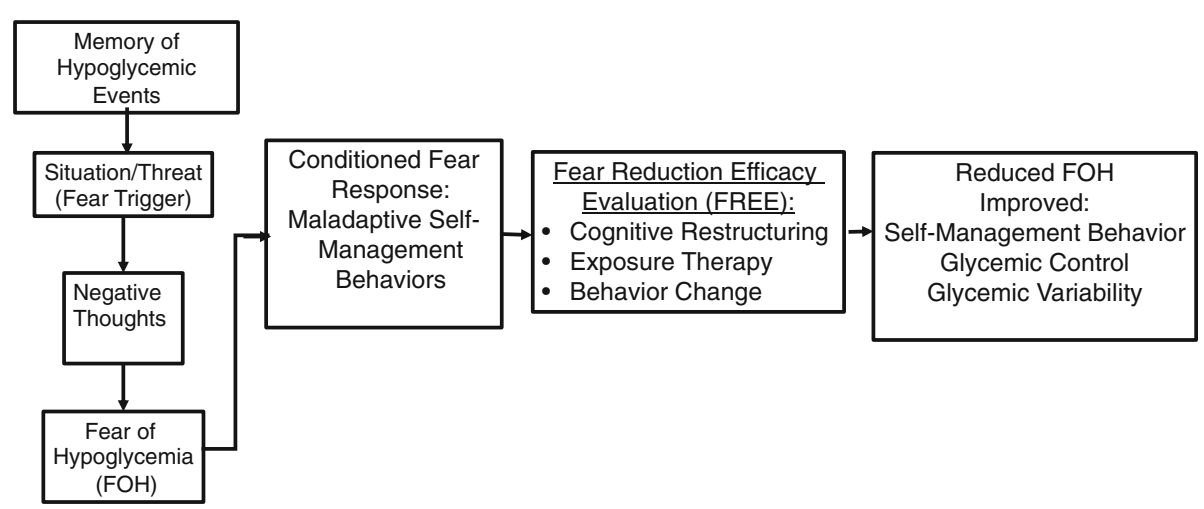

Fig. 2 Cognitive-behavioral model of FREE 
Primary hypothesis: Young adults with T1D participating in the FREE program will demonstrate improvement in $\mathrm{FOH}$ compared to the attention control group.

Secondary hypothesis: Young adults with T1D participating in the FREE program will have improved selfmanagement, glycemic control, and glycemic variability (GV) compared to the attention control group.

\section{Methods/design}

A randomized controlled design using parallel groups will be used to test the study hypotheses. Institutional Review Board approval was obtained (April, 2018) prior to initiating the study from the University of Illinois Office of the Protection of Human Subjects.

\section{Study population and recruitment}

Eligible participants will include young adults (18-35 years) with $\mathrm{T} 1 \mathrm{D} \geq 1$ year, receiving care from an endocrinologist, experiencing $\mathrm{FOH}$ (screening questionnaire) [53], who have previously attended a basic diabetes educational program. Exclusion criteria are pregnancy or breastfeeding, receiving psychotherapy for $\mathrm{FOH}$, and having a co-existing chronic illness or receiving medications (excluding insulin) that may influence diabetes self-management or GV.

Participants will be recruited through two medical centers and local diabetes organizations and websites in the midwestern United States. Informed consent will be obtained from each study participant by a trained member of the study team. Those who meet study criteria will be scheduled for an appointment for the start of the one-week run-in period (week 0) and apply a RT-CGM device with instructions on its care. Participants will be randomly assigned to the FREE intervention or attention control group. During the study period, participants will continue to receive their usual diabetes care, and their health care providers will continue to care for their diabetes as they normally would.

Participants will be assigned a unique code number. A master list that links the subject identity to the data will be kept by the principal investigator (PI) and stored in a locked office separately from the data. All data will be stored and analyzed by code number. The coded data will be entered into a password-protected computer with a secure server for analysis. Paper copy data will be stored in a locked office.

Criteria for discontinuing participation for a given trial participant include: a) participant request or b) determination by the psychologist/interventionist that continuation of the study would not be appropriate due to confounding psychological issues that become evident during the study. If this occurs, a list of counseling resources will be provided.

\section{Sample size determination}

The sample size estimate will allow examination of within-group and between-group differences from beginning to end of the intervention and to calculate effect sizes to power a future larger study. To determine the sample size to meet these goals, the mean, SD, and mean/SD of FOH levels from our pilot studies were used to estimate the expected effect size [48]. The expected estimated effect size was 0.72 . Twenty-five subjects will be required in each group (treatment and control; $n=50$ total). A $20 \%$ attrition rate is expected, based on previous psychoeducational interventions in T1D [39, 41, 43]. Thus, we will accrue 30 subjects per group, to achieve a final sample size of 25 subjects per group ( $n=50$ total).

\section{Randomization and masking}

Randomization will be computer-generated (using REDCap) with permuted blocks in multiples of two and stratified by gender. The randomization process will be overseen by the study statistician $(\mathrm{CP})$. The treatment allocation will be concealed until the time of randomization. Due to the nature of the intervention, participants and their respective interventionists will be aware of their treatment allocation. The project manager will also be aware of the treatment allocation; all other study staff will be masked. Study participants will enter questionnaire data for the primary study outcome directly into REDCap. Secondary outcomes are objectively measured, and those who will analyze these outcomes will be masked to treatment allocation until study completion. At the baseline visit, the project manager and study participant will be informed of the treatment allocation through REDCap. The project manager will then coordinate the first intervention visit with the appropriate interventionist. If an adverse event occurs, unmasking would be permissible.

\section{Interventions}

Participants randomized to the FREE intervention group will: (1) attend eight individual weekly one-hour sessions based on principles of CBT and exposure treatment (Table 1). Treatment will target incorrect beliefs about hypoglycemia, hypervigilance to symptoms, fear of symptoms, and maladaptive behavioral responses in response to glucose levels. Participants will create a fear and avoidance hierarchy and be taught to begin approaching previously feared situations (e.g., spending time alone, reducing snacking, allowing glucose readings to reach lower safe levels, etc.) to experience habituation and the resulting decrease in anxiety. Weekly homework will be assigned to reinforce the content. (2) FREE intervention subjects will continue to wear an unblinded RTCGM for the 8 weeks and (3) complete a daily FOH diary. RT-CGM readings and daily diaries will serve as 
Table 1 Elements of the FREE program

\begin{tabular}{ll}
\hline Elements of the FREE Program & Week 1 \\
\hline Hypoglycemia and its causes & \\
Fear as a normal human emotion & \\
Effect of fear on health and health behaviors & Week 2 \\
Safety and avoidance behaviors & \\
Blood glucose cues & Week 3 \\
Introduction to CBT & \\
$\begin{array}{l}\text { Cognitive restructuring, safety behaviors } \\
\text { Introduction to progressive relaxation }\end{array}$ & Week 4 \\
$\begin{array}{l}\text { Exposure therapy: develop fear hierarchy } \\
\text { Coping strategies }\end{array}$ & Weeks 5- \\
$\begin{array}{l}\text { Exposure practice, coping, relaxation, and cognitive } \\
\text { restructuring } \\
\text { Review techniques learned, develop a plan to maintain }\end{array}$ & Week 8 \\
gains &
\end{tabular}

feedback cues for glucose and FOH levels as part of the FREE program.

Participants randomized to attention control will attend eight weekly individual one-hour sessions on diabetes selfmanagement education (Table 2). Topics follow the American Diabetes Association DSME standards [54] and will be led by a certified diabetes educator (CDE). Weekly homework will be assigned. Participants will continue to wear an unblinded RT-CGM for the 8-week session (for GV measurement only) but will not keep a diary.

\section{Measures}

Data will be collected (1) at the beginning of a one-week run-in period (week 0); (2) at week 4; (3) upon completion of the intervention (week 8); and (4) post-program (week 12). FOH, self-management, and $\mathrm{A1C}$ will be measured, and RT-CGM recordings will be analyzed to determine within-group and between-group differences (Fig. 3).

Table 2 Elements of the attention control (DSME) program

\begin{tabular}{lr}
\hline Elements of the attention control (DSME) program & \\
\hline Living with type 1 diabetes & Week 1 \\
Using insulin safely: blood glucose monitoring & Week 2 \\
$\begin{array}{l}\text { Healthy eating: incorporating nutritional management } \\
\text { into lifestyle }\end{array}$ & Week 3 \\
Healthy eating: carbohydrate counting & Week 4 \\
$\begin{array}{l}\text { Being active: incorporating physical activity into } \\
\text { lifestyle }\end{array}$ & Week 5 \\
Healthy sleep & Week 6 \\
Diabetes in the workplace & Week 7 \\
Preventing complications & Week 8 \\
Review and wrap-Up & \\
\hline
\end{tabular}

Baseline and post-intervention measures

Self-report instruments will be used to obtain demographic, literacy, and health information and previous history of hypoglycemia and diabetes self-management (see Table 3 for a complete list of measures). The scales chosen have strong psychometric properties and have been validated in diabetes populations. The primary outcome, FOH, will be measured using the Worry Subscale of the Hypoglycemia Fear Scale II. This 18item, five-point Likert scale measures situationspecific worries about hypoglycemia and provides one overall score for hypoglycemic worry. The scale has strong psychometric properties (Cronbach's alpha 0.95) [53]. Self-management will be measured with the Diabetes Self-Management Scale (Cronbach's alpha 0.84) [56]. Convergent and construct validity are demonstrated for both scales [53, 56]. Glycemic control will be measured with a fingerstick for A1C using previously published methods (A1C Now ${ }^{\circ}$, Chek Diagnostics, Indianapolis, IN, USA) [48].

\section{Glycemic variability}

Subcutaneous interstitial glucose levels will be monitored using Dexcom G-series continuous glucose monitoring systems ${ }^{\odot}$ (San Diego, CA). To analyze GV, the frequency and time spent in hypo- and hyperglycemia and time in range will be calculated (percentage and minutes; $<70$ and $>180 \mathrm{mg} / \mathrm{dL}$ ). GV will be determined by calculating the daily glucose standard deviation (GlucSD), continuous net glycemic action (CONGA), coefficient of variation $(\mathrm{CV})$, and interquartile range (IQR) $[57,58]$.

\section{Related variables}

Self-efficacy, anxiety, diabetes distress, depressive mood, and quality of life will be measured with validated instruments (Self-Efficacy for Diabetes Scale [59], General Anxiety Disorder-7 Item [GAD-7] [60], Diabetes Distress Scale [61], Center for Epidemiological Studies Depression Scale [CES-D] [62], and Ferrans and Powers Quality of Life Index-Diabetes [55], respectively, at weeks 0,8 , and 12).

\section{Data safety monitoring}

A data safety monitoring committee (DSMC) will be formed to evaluate accumulated data for participation safety, study conduct, and progress. The DSMC will be comprised of an interdisciplinary team of professors from the University of Illinois at Chicago and will be independent of the study sponsor. The DSMC will submit an annual report to the study team, University Institutional Review Board, and the study sponsor. 


\begin{tabular}{|c|c|c|c|c|c|c|c|c|c|c|c|}
\hline \multicolumn{12}{|c|}{ STUDY PERIOD } \\
\hline Week & -1 & 0 & 1 & 2 & 3 & 4 & 5 & 6 & 7 & 8 & 12 \\
\hline Eligibility Screen & $\mathrm{x}$ & & & & & & & & & & \\
\hline Informed consent & & $x$ & & & & & & & & & \\
\hline $\begin{array}{l}\text { Baseline } \\
\text { assessments } \\
\text { (Table 3) }\end{array}$ & & $x$ & & & & & & & & $x$ & $x$ \\
\hline $\mathrm{FOH}$ (outcome) & & $x$ & & & & $\mathrm{x}$ & & & & $x$ & $x$ \\
\hline $\begin{array}{l}\text { Glycemic } \\
\text { variability (RT= } \\
\text { CGM) }\end{array}$ & & $\mathrm{x}$ & $\mathrm{x}$ & $x$ & $x$ & $\mathrm{x}$ & $x$ & $x$ & $\mathrm{x}$ & $x$ & $x$ \\
\hline Allocation & & $x$ & & & & & & & & & \\
\hline FREE sessions & & & $x$ & $x$ & $x$ & $x$ & $x$ & $x$ & $\mathrm{x}$ & $\mathrm{x}$ & \\
\hline $\begin{array}{l}\text { Attention control } \\
\text { sessions }\end{array}$ & & & $x$ & $x$ & $x$ & $x$ & $x$ & $x$ & $x$ & $\mathrm{x}$ & \\
\hline
\end{tabular}

Fig. 3 Spirit figure

\section{Statistical analysis}

The statistical estimation method for this study is a mixed-effects model with repeated measures (SPSS).

Aim 1: Feasibility will be evaluated by assessing recruitment, retention, and participant evaluation. Records will be kept of the number of recruited, screened, eligible, and consented subjects. Retention will be evaluated by weekly attendance (percentage session attendance, program completion rates). Acceptability will be determined through participant evaluation (written evaluation and interview at program completion and a convened advisory group of previous participants). As part of feasibility, we will also track the number of RT-CGM sensor failures, placement sites, time to failure, and adverse sensor site problems.

Aim 2: We will evaluate the effects of within-group and between-group differences from baseline (week 0 ) to program completion (week 8 ) and post program (week

Table 3 Measures

\begin{tabular}{|c|c|c|}
\hline Variables & Measure & Frequency \\
\hline Demographic, health, and literacy information & $\begin{array}{l}\text { Demographic, health questionnaire, Hypoglycemia Patient Questionnaire [2] } \\
\text { Health Literacy Screener (Newest Vital Sign) [55] }\end{array}$ & Week 0 \\
\hline \multicolumn{3}{|l|}{ Aim 1} \\
\hline Recruitment & Number recruited, screened, eligible, consented & Weekly \\
\hline Retention & Attendance rate; completion rate & Weekly \\
\hline \multirow[t]{2}{*}{ Acceptability } & Participant evaluation survey and interview (Appendix P4) & Week 8 \\
\hline & Advisory panel & End of study \\
\hline \multicolumn{3}{|l|}{ Aim 2} \\
\hline $\mathrm{FOH}$ & Hypoglycemia Fear Scale-II (HFS-II [52]) & Weeks $0,4,8,12$ \\
\hline \multicolumn{3}{|l|}{ Glycemic measures } \\
\hline Glycemic control & $\mathrm{A} 1 \mathrm{C}\left(\mathrm{A} 1 \mathrm{C} \mathrm{Now}^{\oplus}\right)$ & Weeks 0, 8, 12 \\
\hline Glycemic variability & $\begin{array}{l}\left.\text { RT-CGM (Dexcom }{ }^{\oplus}\right) \text { : daily glucose standard deviation (GlucSD), continuous } \\
\text { net glycemic action (CONGA), coefficient of variation (CV\%), interquartile } \\
\text { range (IQR), time spent in hypo- and hyperglycemia and time in range }\end{array}$ & Weeks $0-8$ \\
\hline Diabetes self-management & Diabetes Self-Management Questionnaire [54] & Weeks 0, 8, 12 \\
\hline
\end{tabular}


12) on the outcomes $\mathrm{FOH}$, self-management, glycemic control, and GV, using an intent-to-treat approach. To address sex as a biologic variable, sex differences in primary and secondary outcomes will be explored. Diabetes duration and depressive mood will also be statistically controlled.

Hypothesis 1: FOH will be reduced. Within-group and between-group differences in HFS worry score from baseline to study completion and post program will be compared using a mixed-effects model.

Hypothesis 2a: Diabetes self-management will be improved. Within- and between-group differences in the Diabetes Self-Management Scale score from baseline to study completion and post-program will be compared using a mixed-effects model.

Hypothesis 2b: Glycemic control. Within- and between-group differences in $\mathrm{A} 1 \mathrm{C}$ from baseline to study completion and post-program will be compared using a mixed-effects model.

Hypothesis 2c: GV will decrease. GV will be determined through GlucSD, CONGA, CV\%, and IQR, as well as daily time spent in hypo- or hyperglycemia. Within- and between-group differences will be calculated from daily RT-CGM recordings using a mixedeffects model.

\section{Management of missing data}

Missing values will be analyzed to better understand the characteristics of missing patterns. Once the missing patterns are understood, an imputation method will be determined if appropriate. As this is a pilot study, missing value information will be used to plan the next stage and larger study.

\section{Participant retention strategy}

Both FREE intervention and attention control groups will (1) have weekly sessions scheduled at a time convenient for the participants; (2) wear a RT-CGM; (3) receive appointment reminders; (4) be compensated at weeks 4,8 , and 12; and (5) receive a personalized folder with copies of their CGM recordings at study's end. Weekly sessions for both the FREE and attention control groups will include topics of interest and value to the study population. Use of RT-CGM technology is highly desirable for many young adults with T1D and served as both an incentive and retention factor in our previous studies [48].

\section{Intervention fidelity}

To maintain treatment fidelity, both FREE and attention control sessions will follow a manualized protocol. Also, sessions will be audio-recorded and reviewed for fidelity to the treatment protocol by study staff.

\section{Discussion}

This study protocol directly focuses on $\mathrm{FOH}$ reduction. This study will generate information for a larger clinical trial to test the effectiveness of the FREE intervention compared to diabetes management education to reduce $\mathrm{FOH}$, improve self-management behavior, and improve glycemic control and GV. If effective, this intervention will serve as an important adjunct to diabetes care in young adults with T1D, reduce the development of diabetes complications, and improve quality of life.

\section{Trial status}

This study is in the recruitment phase (protocol version 7, 5/17/2019). Recruitment began November 2018. The first participant was enrolled January 2019. Recruitment is expected to be completed by June 2020 .

\section{Supplementary information}

Supplementary information accompanies this paper at https://doi.org/10. 1186/s13063-019-3876-4

Additional file 1. SPIRIT 2013 checklist

\section{Abbreviations}

A1C: Glycated hemoglobin; CBT: Cognitive behavioral therapy; CES-D: Center for Epidemiological Studies Depression Scale; CGM: Continuous glucose monitoring; CONGA: Continuous net glycemic action; CV: Coefficient of variation; FOH: Fear of hypoglycemia; FREE: Fear reduction efficacy evaluation; GAD: General Anxiety Disorder Scale; GlucSD: Glucose standard deviation; GV: Glycemic variability; HFS-II: Hypoglycemia Fear Scale; IQR: Interquartile range; QOL: Quality of life; REDCap: Research Electronic Data Capture; RT-CGM: Real-time continuous glucose monitoring; T1D: Type 1 diabetes

\section{Acknowledgement}

This study is funded by the National Institutes of Health, National Institute of Diabetes and Digestive and Kidney Diseases (R21DK116146). We would like to thank the University of Illinois CCTS Biomedical Informatics Core (UL1TR002003) for assistance with recruitment and Kevin Grandfield, Publication Manager of the University of Illinois Chicago Department of Biobehavioral Health Science, for editorial assistance. The sponsor /funders played no part in study design; collection, management, and analysis plan for the data; writing of this report; and the decision to submit the report for publication.

\section{Authors' contributions}

All authors (PMN, JD, LQ, CP, DM, SP) contributed to the study concept, design, and procedures. CP created the statistical analysis plan and randomization sequence. JD and SQ contributed to the intervention content. PMN drafted the manuscript, and all other authors contributed to the final version. All authors read and approved the final manuscript.

Availability of data and materials Not applicable at this time.

Ethics approval and consent to participate

Ethics approval and consent to participate: the Institutional Review Board of the University of Illinois at Chicago (Office of the Protection of Human Subjects, Board \#3) approval was obtained prior to initiating the study. IRB Protocol \# 2018-0382. All participants provided informed consent prior to study participation.

Consent for publication Not applicable. 


\section{Competing interests}

All authors declare that they have no competing interests.

\section{Author details}

'Department of Biobehavioral Health Science, University of Illinois at Chicago College of Nursing, 845 South Damen Avenue (MC 802), Chicago, IL 60612,

USA. ${ }^{2}$ Department of Psychiatry, University of Illinois at Chicago, Chicago, IL, USA. ${ }^{3}$ Department of Health Systems Science, University of Illinois at Chicago College of Nursing, Chicago, IL, USA. ${ }^{4}$ Endocrinology/Diabetes and Metabolism, Department of Medicine, University of Illinois at Chicago, Chicago, IL, USA. ${ }^{5}$ Loyola University Chicago, Chicago, IL, USA.

Received: 29 August 2019 Accepted: 2 November 2019 Published online: 30 December 2019

\section{References}

1. Davis $\mathrm{S}$, Alonso MD. Hypoglycemia as a barrier to glycemic control. J Diabetes Complicat. 2004;18(1):60-8.

2. Seaquist ER, Anderson J, Childs B, et al. Hypoglycemia and diabetes: a report of a workgroup of the American Diabetes Association and the Endocrine Society. Diabetes Care. 2013;36(5):1384-95.

3. Cryer PE. Death during intensive glycemic therapy of diabetes: mechanisms and implications. Am J Med. 2011;124(11):993-6.

4. Lawton J, Rankin D, Cooke DD, Elliott J, Amiel S, Heller S. Self-treating hypoglycaemia: a longitudinal qualitative investigation of the experiences and views of people with type 1 diabetes. Diabet Med. 2013;30(2):209-15

5. Polonsky WH, Davis CL, Jacobson AM, Anderson BJ. Correlates of hypoglycemic fear in type I and type II diabetes mellitus. Health Psychol. 1992;11(3):199-202.

6. Irvine AA, Cox D, Gonder-Frederick L. Fear of hypoglycemia: relationship to physical and psychological symptoms in patients with insulin-dependent diabetes mellitus. Health Psychol. 1992;11(2):135-8.

7. Cox DJ, Gonder-Frederick L, Antoun B, Clarke W, Cryer P. Psychobehavioral metabolic parameters of severe hypoglycemic episodes. Diabetes Care. 1990;13(4):458-9.

8. Currie CJ, Morgan CL, Poole CD, Sharplin P, Lammert M, McEwan P. Multivariate models of health-related utility and the fear of hypoglycaemia in people with diabetes. Curr Med Res Opin. 2006;22(8):1523-34.

9. Bohme P, Bertin E, Cosson E, Chevalier N. Fear of hypoglycaemia in patients with type 1 diabetes: do patients and diabetologists feel the same way? Diabetes Metab. 2013;39(1):63-70.

10. Davis M, Walker DL, Miles L, Grillon C. Phasic vs sustained fear in rats and humans: role of the extended amygdala in fear vs anxiety. Neuropsychopharmacology. 2010;35(1):105-35.

11. Green L, Feher M, Catalan J. Fears and phobias in people with diabetes. Diabetes Metab Res Rev. 2000;16(4):287-93.

12. Adolphs R. The biology of fear. Curr Biol. 2013;23(2):R79-93.

13. Boyle S, Allan C, Millar K. Cognitive-behavioural interventions in a patient with an anxiety disorder related to diabetes. Behav Res Ther. 2004:42(3): 357-66

14. Kilgus MD, Ward NG, Maxmen JS. Essential psychopathology and its treatment. New York: W.W. Norton; 2009.

15. Brazeau AS, Rabasa-Lhoret R, Strychar I, Mircescu H. Barriers to physical activity among patients with type 1 diabetes. Diabetes Care. 2008;31(11): 2108-9.

16. Leiter LA, Yale JF, Chiasson JL, Harris S, Kleinstiver P, Sauriol L. Assessment of the impact of fear of hypoglycemic episodes on glycemic and hypoglycemic management. Can J Diabetes. 2005;29(3):1-7.

17. Goebel-Fabbri AE, Fikkan J, Franko DL, Pearson K, Anderson BJ, Weinger K. Insulin restriction and associated morbidity and mortality in women with type 1 diabetes. Diabetes Care. 2008:31(3):415-9.

18. Hanna KM, Weaver MT, Stump TE, Slaven JE, Fortenberry JD, DiMeglio LA. Readiness for living independently among emerging adults with type 1 diabetes. Diabetes Educ. 2013;39(1):92-9.

19. Weiner MF, Skipper FP Jr. Euglycemia: a psychological study. Int J Psychiatry Med. 1978:9(3-4):281-7.

20. Zander H, Beneke J, Veagedes D, Muller A, Zwaan M. Type 1 diabetes mellitus and bulimia nervosa: insulin restriction does not always represent purging of unwanted calories. Pract Diab Int. 2014;31(4):161-163a.
21. Gonzalez JS, Tanenbaum ML, Commissariat PV. Psychosocial factors in medication adherence and diabetes self-management: Implications for research and practice. Am Psychol. 2016;71(7):539-51.

22. Anderbro T, Amsberg $S$, Adamson U, et al. Fear of hypoglycaemia in adults with Type 1 diabetes. Diabet Med. 2010;27(10):1151-8.

23. Richmond J. Effects of hypoglycaemia: patients' perceptions and experiences. Br J Nurs. 1996;5(17):1054-9.

24. Desjardins K, Brazeau AS, Strychar I, Rabasa-Lhoret R. Are bedtime nutritional strategies effective in preventing nocturnal hypoglycaemia in patients with type 1 diabetes? Diabetes. Obes Metab. 2014;16(7):577-87.

25. Miller KM, Foster NC, Beck RW, et al. Current state of type 1 diabetes treatment in the U.S.: updated data from the T1D Exchange clinic registry. Diabetes Care. 2015;38(6):971-8.

26. Anderson BJ, Wolpert HA. A developmental perspective on the challenges of diabetes education and care during the young adult period. Patient Educ Couns. 2004;53(3):347-52.

27. Garvey KC, Markowitz JT, Laffel LM. Transition to adult care for youth with type 1 diabetes. Curr Diabetes Rep. 2012;12(5):533-41.

28. Barnard KD, Parkin C, Young A, Ashraf M. Use of an automated bolus calculator reduces fear of hypoglycemia and improves confidence in dosage accuracy in patients with type 1 diabetes mellitus treated with multiple daily insulin injections. J Diabetes Sci Technol. 2012;6(1):144-9.

29. Barnard KD, Skinner TC. Cross-sectional study into quality of life issues surrounding insulin pump use in type 1 diabetes. Pract Diabetes Int. 2008; 25(5):194-200.

30. Davey RJ, Stevens K, Jones TW, Fournier PA. The effect of short-term use of the Guardian RT continuous glucose monitoring system on fear of hypoglycaemia in patients with type 1 diabetes mellitus. Prim Care Diabetes. 2012;6(1):35-9.

31. Hermanides J, Norgaard K, Bruttomesso D, et al. Sensor-augmented pump therapy lowers $\mathrm{HbA}$ (1c) in suboptimally controlled Type 1 diabetes; a randomized controlled trial. Diabet Med. 2011:28(10):1158-67.

32. Nicolucci A, Maione A, Franciosi M, et al. Quality of life and treatment satisfaction in adults with Type 1 diabetes: a comparison between continuous subcutaneous insulin infusion and multiple daily injections. Diabet Med. 2008:25(2):213-20.

33. Rubin RR, Peyrot M. Health-related quality of life and treatment satisfaction in the Sensor-Augmented Pump Therapy for A1C Reduction 3 (STAR 3) trial. Diabetes Technol Ther. 2012;14(2):143-51.

34. Schmitz KH, Jacobs DR, Hong C-P, Steinberger J, Moran A, Sinaikko AR. Association of physical activity with insulin sensitivity in children. Int J Obes Relat Metab Disord. 2002;26(10):1310-6.

35. Costea M, lonescu-Tirgoviste C, Cheta D, Mincu I. Fear of hypoglycemia in type 1 (insulin-dependent) diabetic patients. Revue Roumaine Medecine Interne. 1993;31(4):291-5.

36. Cox D, Gonder-Frederick L, Polonsky W, Schlundt D, Julian D, Clarke W. A multicenter evaluation of blood glucose awareness training-II. Diabetes Care. 1995;18(4):523-8

37. Cox DJ, Gonder-Frederick L, Polonsky W, Schlundt D, Kovatchev B, Clarke W. Blood glucose awareness training (BGAT-2): long-term benefits. Diabetes Care. 2001;24(4):637-42.

38. Cox DJ, Ritterband L, Magee J, Clarke W, Gonder-Frederick L. Blood glucose awareness training delivered over the internet. Diabetes Care. 2008:31(8):1527-8.

39. George JT, Valdovinos AP, Russell I, et al. Clinical effectiveness of a brief educational intervention in Type 1 diabetes: results from the BITES (Brief Intervention in Type 1 diabetes, Education for Self-efficacy) trial. Diabet Med. 2008;25(12):1447-53.

40. Ismail K, Thomas SM, Maissi E, et al. Motivational enhancement therapy with and without cognitive behavior therapy to treat type 1 diabetes: a randomized trial. Ann Intern Med. 2008;149(10):708-19.

41. Schachinger $H$, Hegar K, Hermanns $N$, et al. Randomized controlled clinical trial of Blood Glucose Awareness Training (BGAT III) in Switzerland and Germany. J Behav Med. 2005;28(6):587-94.

42. Rondags SM, de Wit M, Twisk JW, Snoek FJ. Effectiveness of HypoAware, a brief partly web-based psychoeducational intervention for adults with type 1 and insulin-treated type 2 diabetes and problematic hypoglycemia: a cluster randomized controlled trial. Diabetes Care. 2016;39(12):2190-6.

43. Amsberg $\mathrm{S}$, Anderbro T, Wredling $\mathrm{R}$, et al. A cognitive behavior therapybased intervention among poorly controlled adult type 1 diabetes patients--a randomized controlled trial. Patient Educ Couns. 2009:77(1):72-80. 
44. Kilpatrick ES, Rigby AS, Goode K, Atkin SL. Relating mean blood glucose and glucose variability to the risk of multiple episodes of hypoglycaemia in type 1 diabetes. Diabetologia. 2007;50(12):2553-61.

45. Quagliaro L, Piconi L, Assaloni R, Martinelli L, Motz E, Ceriello A. Intermittent high glucose enhances apoptosis related to oxidative stress in human umbilical vein endothelial cells: the role of protein kinase $\mathrm{C}$ and $\mathrm{NAD}(\mathrm{P}) \mathrm{H}-$ oxidase activation. Diabetes. 2003;52(11):2795-804.

46. Ceriello A, Novials A, Ortega E, et al. Evidence that hyperglycemia after recovery from hypoglycemia worsens endothelial function and increases oxidative stress and inflammation in healthy control subjects and subjects with type 1 diabetes. Diabetes. 2012;61(11):2993-7.

47. Soupal J, Skrha J Jr, Fajmon M, et al. Glycemic variability is higher in type 1 diabetes patients with microvascular complications irrespective of glycemic control. Diabetes Technol Ther. 2014;16(4):198-203.

48. Martyn-Nemeth P, Quinn L, Penckofer S, Park C, Hofer V, Burke L. Fear of hypoglycemia: Influence on glycemic variability and self-management behavior in young adults with type 1 diabetes. J Diabetes Complicat. 2017; 31(4):735-41.

49. Kildegaard J, Christensen TF, Hejlesen OK. Sources of glycemic variability--what type of technology is needed? J Diabetes Sci Technol. 2009;3(4):986-91.

50. Hartley CA, Phelps EA. Changing fear: the neurocircuitry of emotion regulation. Neuropsychopharmacology. 2010;35(1):136-46.

51. Beck JS. Cognitive behavior therapy. 2nd ed. New York: The Guilford Press; 2011.

52. Beck J, Greenwood DA, Blanton L, et al. 2017 National standards for diabetes self-management education and support. Diabetes Care. 2017; 40(10):1409-19.

53. Gonder-Frederick LA, Schmidt KM, Vajda KA, et al. Psychometric properties of the hypoglycemia fear survey-ii for adults with type 1 diabetes. Diabetes Care. 2011;34(4):801-6.

54. Haas L, Maryniuk M, Beck J, Cox C, Duker, et al. National standards for diabetes self-management education and support. Diabetes Care. 2012;35:2393-401.

55. Timar R, Velea I, Timar B, et al. Factors influencing the quality of life perception in patients with type 2 diabetes mellitus. Patient Prefer Adherence. 2016;10:2471-7.

56. Schmitt A, Gahr A, Hermanns N, Kulzer B, Huber J, Haak T. The Diabetes Self-Management Questionnaire (DSMQ): development and evaluation of an instrument to assess diabetes self-care activities associated with glycaemic control. Health Qual Life Outcomes. 2013;11:138.

57. Rodbard D. New and improved methods to characterize glycemic variability using continuous glucose monitoring. Diabetes Technol Ther. 2009;11(9): 551-65.

58. Bergenstal RM, Ahmann AJ, Bailey T, et al. Recommendations for standardizing glucose reporting and analysis to optimize clinical decision making in diabetes: the Ambulatory Glucose Profile (AGP). Diabetes Technol Ther. 2013;15(3):198-211.

59. Lorig K. Self-efficacy for diabetes. https://www.selfmanagementresource. com/docs/pdfs/English___self-efficacy_diabetes.pdf, accessed 31 Oct 2019.

60. Jordan P, Shedden-Mora MC, Lowe B. Psychometric analysis of the Generalized Anxiety Disorder scale (GAD-7) in primary care using modern item response theory. PLoS One. 2017;12(8):e0182162.

61. Fisher L, Polonsky WH, Hessler DM, et al. Understanding the sources of diabetes distress in adults with type 1 diabetes. J Diabetes Complicat. 2015;29(4):572-7.

62. Radloff L. The CES-D scale: a self-report depression scale for research in the general population. Appl Psychol Meas. 1977;1:385-401.

63. Yoon JE, Sunwoo JS, Kim JS, et al. Poststroke glycemic variability increased recurrent cardiovascular events in diabetic patients. J Diabetes Complicat. 2017;31(2):390-4.

\section{Publisher's Note}

Springer Nature remains neutral with regard to jurisdictional claims in published maps and institutional affiliations.

\section{Ready to submit your research? Choose BMC and benefit from:}

- fast, convenient online submission

- thorough peer review by experienced researchers in your field

- rapid publication on acceptance

- support for research data, including large and complex data types

- gold Open Access which fosters wider collaboration and increased citations

- maximum visibility for your research: over $100 \mathrm{M}$ website views per year

At BMC, research is always in progress.

Learn more biomedcentral.com/submissions 